Elsevier Editorial System(tm) for Acta Biomaterialia

Manuscript Draft

Manuscript Number: AB-08-767R1

Title: Ocular injectable formulation assessment for oxidized dextran-based hydrogels

Article Type: Full Length Article

Section/Category:

Keywords: Injectable; Hydrogel; Oxidized Dextran; In vitro, Ocular delivery

Corresponding Author: Dr. Maria Helena Gil,

Corresponding Author's Institution: Faculdade de Ciências e Tecnologia da Universidade de Coimbra

First Author: João Maia, MSc

Order of Authors: João Maia, MSc; Maximiano P Ribeiro, Msc; Carla Ventura, PhD; Rui A Carvalho, PhD; Ilídio J Correia, PhD; Maria Helena Gil

Manuscript Region of Origin:

Abstract: Initiator-free injectable hydrogels are very interesting for drug and/or cell delivery applications, since they can be administered in a minimally invasive way, without recurring to chemical initiators that could be harmful. In the current work, oxidized dextran crosslinked (dexOx) with adipic acid dihydrazide (AAD) hydrogels were further characterized and tuned to produce formulations, having in sight an injectable ocular formulation for the possible treatment of posterior eye diseases. The gelation rate and the hydrogels dissolution profile were shown to be dependent on the balance between the dextran oxidation degree, and both components concentration. On the in vitro studies, rabbit corneal endothelial cells were seeded on the hydrogels, to assess cytotoxicity. Hydrogels prepared with low oxidized dextrans were able to promote cell adhesion and 
proliferation to confluence in just 24 hours, while higher oxidized samples promoted cell adhesion and proliferation, but without achieving confluence. Cell viability studies were performed using MTS assays to verify the non-cytotoxicity of hydrogels and their degradation byproducts, rendering these formulations attractive for further in vivo studies. 


\section{Ocular injectable formulation assessment for oxidized}

\section{dextran-based hydrogels}

João Maia ${ }^{\mathrm{a}}$, Maximiano P. Ribeiro ${ }^{\mathrm{b}}$, Carla Ventura ${ }^{\mathrm{a}}$, Rui A. Carvalho ${ }^{\mathrm{c}}$, Ilídio J. Correia ${ }^{\mathrm{b}}$, Maria H.

$$
\mathrm{Gil}^{\mathrm{a} *}
$$

${ }^{\text {a }}$ Departamento de Engenharia Química da Faculdade de Ciências e Tecnologia da Universidade de Coimbra, Rua Sílvio Lima - Pólo II, 3030-790 Coimbra, Portugal; ${ }^{b}$ Centro de Investigação em Ciências da Saúde, Faculdade de Ciências da Saúde, Universidade da Beira Interior, Av. Infante D. Henrique, Covilhã, Portugal; and ${ }^{\mathrm{c}}$ Espectroscopia RMN, Centro de Neurociências e Biologia Celular e Departamento de Bioquímica da Faculdade de Ciências e Tecnologia da Universidade de Coimbra

\footnotetext{
*Corresponding Author: Departamento de Engenharia Química da Faculdade de Ciências e Tecnologia da Universidade de Coimbra, Rua Sílvio Lima - Pólo II, 3030-790 Coimbra, Portugal; Tel.: +351 239798 743; Fax: +351 239798 703; Email address: hgil@eq.uc.pt
} 


\begin{abstract}
Initiator-free injectable hydrogels are very interesting for drug and/or cell delivery applications, since they can be administered in a minimally invasive way, without recurring to chemical initiators that could be harmful. In the current work, oxidized dextran crosslinked (dexOx) with adipic acid dihydrazide (AAD) hydrogels were further characterized and tuned to produce formulations, having in sight an injectable ocular formulation for the possible treatment of posterior eye diseases. The gelation rate and the hydrogels dissolution profile were shown to be dependent on the balance between the dextran oxidation degree, and both components concentration. On the in vitro studies, rabbit corneal endothelial cells were seeded on the hydrogels, to assess cytotoxicity. Hydrogels prepared with low oxidized dextrans were able to promote cell adhesion and proliferation to confluence in just 24 hours, while higher oxidized samples promoted cell adhesion and proliferation, but without achieving confluence. Cell viability studies were performed using MTS assays to verify the non-cytotoxicity of hydrogels and their degradation byproducts, rendering these formulations attractive for further in vivo studies.
\end{abstract}

Keywords: Injectable; Hydrogel; Oxidized Dextran; In vitro; Ocular delivery 


\section{Introduction}

Ocular diseases of the posterior eye segment are the most common cause of visual disorders in industrialized countries ${ }^{1}$. These illnesses include, for example, cataracts, diabetic retinopathy, macular degeneration associated with aging and retinitis pigmentosa ${ }^{1,2}$. The visual disorders cause discomfort, anxiety and fear of losing the vision in patients.

Until now, ocular drug market is dominated by drugs, which were conceived to treat illnesses that affect the anterior eye segment, such medicines include: antibiotics, anti-inflammatory agents or antiglaucoma drugs, usually as eye drop formulations ${ }^{1,3}$. These topical applications, in the form of eye drops, are ineffective in many cases, due mainly to the drainage system of the eye, which leads to poor ocular bioavailability ${ }^{4}$. New drugs have been developed to reach the posterior eye segment, although, most of them are administered through repeated intravitreal injections. This method is associated with complications such as pain, increased intraocular pressure, retinal detachment and endophtalmitis (which may lead to blindness). New methods of drug delivery, more secure, efficient, comfortable and with prolonged activity are needed, to minimize the number of injections. Currently, there are systems of controlled drug delivery on the market or being tested, such as implants, hydrogels and colloids ${ }^{1}$.

Hydrogels are hydrophilic polymer networks, which may absorb up to thousands of times their dry weight in water. Hydrogels may be chemically stable or they may degrade and eventually disintegrate and dissolve. They are called "reversible" or "physical" gels when the networks are held together by molecular entanglements, and/or secondary forces including ionic, H-bonding or hydrophobic forces. Hydrogels are called "permanent" or "chemical" gels when they are covalently-crosslinked networks ${ }^{5}$.

Recently, Van Tomme and collaborators compiled the different strategies used in producing dextran-based hydrogels, demonstrating dextran as a very versatile starting polymer for hydrogel synthesis $^{6}$. Furthermore, pharmaceuticals and various potential therapeutic agents can easily be incorporated and its release profile controlled. Recently, human embryonic stem-cell encapsulation was also successfully achieved in bioactive hydrogels of dextran-acrylate ${ }^{7}$. 
Dextran is biocompatible and can be degraded through the action of dextranases in various organs in the human body, including liver, spleen, kidney and colon ${ }^{8}$.

Dextran oxidation by sodium periodate is an easy and well-known way to functionalize dextran with aldehyde moieties ${ }^{9}$. This chemical functionality has been widely tested to conjugate N-nucleophiles, due to their fast and almost complete reaction ${ }^{10}$. This approach has been tested on the synthesis of prodrugs $^{11}$, as a spacer in enzyme immobilization ${ }^{12}$ or for growth factor controlled release $\mathrm{e}^{13}$. On the preparation of hydrogels, different aminated crosslinkers, like chitosan ${ }^{14,15}$, gelatin ${ }^{16}, 8$-arm PEG amine $^{17}$ or polyhydrazides ${ }^{18}$ have been used to yield chemical initiator-free formulations.

Recently, we described an injectable formulation composed of oxidized dextran (dexOx) crosslinked with adipic acid dihydrazide (AAD) ${ }^{19}$. The dexOx with $15 \%$ oxidation degree (OD) was crosslinked with AAD, forming a gel within 2-4 minutes, depending on the AAD concentration used. The obtained hydrogels were characterized by their mechanical properties $(7-32 \mathrm{kPa})$, swelling and degradation (9 to 23 days) behavior under physiologic conditions.

In this work, oxidized dextran, with various OD, was studied in order to improve the control of the system properties. The influence of dextran OD and concentration in the solution viscosity was monitored. We observed that the hydrogel swelling and dissolution could also be controlled by the dextran oxidation degree and that the dissolution profile could be extended for more than 2 months, improving the previous studied formulation. Cell toxicity assays were carried out in 96-well plates with different dexOx's hydrogels. Cell viability studies were performed using rabbit corneal endothelial cells, which were seeded on top of the dexOx's hydrogels. The cell adhesion and growth was visualized by optical microscopy and dehydrogenase activity of cells was evaluated by reduction of the MTS reagent.

\section{Experimental Section}




\subsection{Materials}

Dextran (from Leuconostoc mesenteroides; $\mathrm{M}_{\mathrm{w}} 60^{\prime} 000$ Da, according to Fluka's specification), sodium periodate, adipic acid dihydrazide, tert-butyl carbazate (tBC), ethyl carbazate (EtC), phosphate buffered saline (PBS), dialysis tubes (MWCO $\sim 12^{\prime} 000 \mathrm{Da}$ ), amphotericin B, L-glutamine, Minimum Essential Medium Eagle, penicillin G, streptomycin, and trypsin were purchased from Sigma (Sintra, Portugal). Foetal bovine serum was purchased from Biochrom AG (Berlin, Germany). The 3-[4,5dimethylthiazol-2-yl]-5-(3-carboxymethoxyphenyl)-2-(4-sulfophenyl)-2H-tetrazolium, inner $\quad$ salt (MTS) and electron coupling reagent (phenazine methosulfate; PMS) were purchased from Promega. T-flasks and 96 well plates were purchased from Nunc (Denmark).

\subsection{Dextran oxidation}

An aqueous solution of dextran $(1 \mathrm{~g} ; 0.125 \%$, w/v) was oxidized with $2 \mathrm{~mL}$ of sodium periodate solution with different concentrations $(33-264 \mathrm{mg} / \mathrm{mL})$ to yield theoretical oxidations from 5 to $40 \%$, at room temperature. The reaction was stopped after 4 hours. The resulting solution was dialyzed for 3 days against water, using a dialysis tube with a MWCO 12-14,000 Da, and then lyophilized (Snidjers Scientific type 2040, Tillburg, Holland). The scale-up of the reaction was done using the same procedure albeit using $30 \mathrm{~g}$ of dextran and a calculated amount of periodate to yield a theoretical oxidation of $5,10,25$ and $40 \%$.

\subsection{Nuclear magnetic resonance (NMR) and size exclusion chromatography (SEC)}

The OD of dexOx is defined as the number of oxidized residues per 100 glucose residues (OD refers to the theoretical value unless otherwise stated) and quantified by using $\mathrm{tBC}^{20,21}$ and $\mathrm{EtC}$. The carbazates react with aldehyde groups to form carbazones in the same way hydrazones are formed in the presence of hydrazides.

${ }^{1} \mathrm{H}$ spectra were acquired on a Varian 600 NMR spectrometer (Palo Alto, CA) using a $3 \mathrm{~mm}$ broadband NMR probe. ${ }^{1} \mathrm{H}$ NMR spectra were recorded in $\mathrm{D}_{2} \mathrm{O}(20-25 \mathrm{mg}$ in $0.2 \mathrm{~mL}$; pD of ca. 5.0) 
using a $90^{\circ}$ pulse and a relaxation delay of $30 \mathrm{~s}$. The water signal, used as reference line, was set at $\delta$ $4.75 \mathrm{ppm}$ and was partially suppressed by irradiation during the relaxation delay. A total of 32 scans were acquired for each ${ }^{1} \mathrm{H}$ NMR spectra. The spectra were analyzed with iNMR software, version 2.6.4 (www.inmr.net).

Size exclusion chromatography was performed in a HPLC system composed of a degasser and a WellChrom Maxi-Star k-1000 pump (Knauer), coupled to a LS detector (evaporative light scattering PL-EMD 960) and one column (PL aquagel-OH Mixed $8 \mu \mathrm{m}$ ) from Polymer Laboratories. The whole system was kept at room temperature and the eluent used was $\mathrm{KNO}_{3}(0.001 \mathrm{M} ; \mathrm{pH}$ 3.9) at a flow rate of $0.4 \mathrm{~mL} / \mathrm{min}$. Samples and standards were dissolved in the eluent at 4-6 mg/mL (Fluka Chemie AG, dextran standards from $12-80 \mathrm{kDa})$.

\subsection{DexOx's solutions viscosity}

Dextran and dexOx's solutions, with concentrations ranging from 10 to $30 \%(\mathrm{w} / \mathrm{w})$ in $\mathrm{PBS}$, were prepared and analyzed in a Brookfield Programmable D-II+ Viscometer with a S18 spindle, assisted by DVLoader v1.0 software. The chamber temperature was controlled by an external bath to $25^{\circ} \mathrm{C}$, and the chamber was loaded with $8 \mathrm{~mL}$ of solution.

\subsection{Hydrogel preparation and characterization}

The several oxidized dextrans were dissolved in different concentrations; dexOx 5\% (D5) and 10\% (D10) solutions were used at 30\% (w/w) and dexOx 25\% (D25) at 20\% (w/w), in aqueous solvent (PBS) at $37^{\circ} \mathrm{C}$ until a liquid solution was obtained and then kept at 5 to $8{ }^{\circ} \mathrm{C}$, until further use. Then, $250 \mu \mathrm{L}$ of a given dexOx solution was mixed with $250 \mu \mathrm{L}$ of a given AAD solution on homemade Teflon ${ }^{\circledR}$ molds and let to crosslink never less than 2 hours (except for the D5 hydrogels, which were let to cure over night). The AAD concentrations used are calculated based on a given molar percentage of dextran residues. The hydrogels nomenclature is as follows: dexOx 5\% + AAD 5\% - D5A5; and so forth. 


\subsection{Dynamic swelling experiments}

DexOx hydrogels, after being prepared and weighted $\left(\mathrm{W}_{\mathrm{i}}\right)$, were immersed in PBS (ca. $\left.5 \mathrm{~mL}\right)$ in 6wells cell culture plates, at $37^{\circ} \mathrm{C}$. At regular time intervals, they were removed from the aqueous solution, blotted in filter paper, weighted $\left(\mathrm{W}_{\mathrm{t}}\right)$ and returned to the original well while PBS was replaced.

$$
\text { Swelling Index: } \quad S I=\frac{W t}{W i}
$$

\subsection{Rheological analysis}

Rheological experiments were carried out using the parallel plate geometry (20 mm diameter, steel) of a Haake Rheostress RS 1. On the calculation of gelation period, both solutions (190 $\mu \mathrm{L}$ each) were mixed on the bottom plate, and the upper plate was positioned at a gap of $1 \mathrm{~mm}$ (after gap optimization). This procedure took around 20 seconds after which the experiment was started at low frequency $(0.5 \mathrm{~Hz})$ and stress $(0.1 \mathrm{~Pa})$, to avoid interference with the network formation. The gelation rate was followed and the gelation period was considered to be the crossover point between G' and $G^{\prime \prime}\left(G^{\prime}=G^{\prime \prime}\right)$.

\subsection{Cell source and growth}

Corneal endothelial cells from rabbit were obtained as previously described ${ }^{22}$. Subsequently, cells were platted in T-flasks of $25 \mathrm{~cm}^{3}$ with Minimum Essential Medium Eagle (MEM) with heatinactivated foetal bovine serum (FBS, $10 \% \mathrm{v} / \mathrm{v}$ ) and growth factors to do primary culture in agreement with the procedures previously described in the literature ${ }^{23}$. T-flasks with cells were incubated in a $5 \%$ carbon dioxide humidified atmosphere at $37^{\circ} \mathrm{C}$. On day 3 , the medium was changed and here after every three days. Six days after cells attained confluence.

After confluence was obtained, cells were sub-cultivated using 5 min incubation in $0.18 \%$ trypsin $(1: 250)$ and $5 \mathrm{mM}$ EDTA. The free cells were added to an equal volume of culture medium. Following 
centrifugation, cells were resuspended in sufficient culture medium and seed in 96 well plates containing the biomaterials.

\subsection{Cell culture and in vitro cytotoxicity studies}

Two crosslinking degrees of AAD for each dexOx were selected and used on cytocompatibility tests. The oxidized dextran samples and AAD were either dissolved in PBS or in the appropriate cell culture medium, MEM.

Each formulation in the form of hydrogel was introduced $(n=6)$, on wells of 96-wells cell culture plates, never exceeding $60 \mu \mathrm{L}$. The plates were irradiated for 30 minutes with $\mathrm{UV}$, before being seeded with cells.

Forth passage endothelial corneal cells were seeded, at a density of 90'000 cells per well, into a 96well plate containing the hydrogels. The plate was incubated at $37^{\circ} \mathrm{C}$, under a $\mathrm{CO}_{2}(5 \%)$ humidified atmosphere. After 1, 3 and 7 days, cell viability was assessed through MTS assays. A CellTiter 96® AQueous Assay composed of 3-[4,5-dimethylthiazol-2-yl]-5-(3-carboxymethoxyphenyl)-2-(4sulfophenyl)-2H-tetrazolium, inner salt (MTS) and an electron coupling reagent (phenazine methosulfate; PMS) from Promega were used. $20 \mu \mathrm{l}$ of MTS/PMS were added to each sample and incubated for $4 \mathrm{~h}$ at $37^{\circ} \mathrm{C}$, under a $\mathrm{CO}_{2}(5 \%)$ atmosphere. The absorbance of the samples was determined at $492 \mathrm{~nm}$ using a Biorad Microplate Reader Benchmark.

Wells containing cells in culture medium without biomaterials were used as negative control (K-). Ethanol $96 \%$ was added to wells containing both types of cells to have a positive control $(\mathrm{K}+)$. The samples were analysed using an Olympus CX41 optical microscope equipped with an Olympus SP$500 \mathrm{UZ}$ digital camera.

\section{Results and Discussion}

\subsection{DexOx characterization}


Following previous work ${ }^{19}$, we characterized hydrogels made from dexOx with a wider range of oxidation degrees, crosslinked with different amounts of AAD. Dextran was oxidized by using sodium periodate at different percentages and characterized by ${ }^{1} \mathrm{H}-\mathrm{NMR}$. The oxidation degree was not estimated by the TNBS assay, due to the good correlation of the NMR titration with the colorimetric assay, as shown in previous work ${ }^{19}$. The tert-butyl carbazate titration, however, causes sample precipitation when reacted with high oxidation degree samples, not allowing NMR spectra acquisition (D40, Table 1). We suggest that this effect is caused by the large tert-butyl moiety and we hypothesized that a less bulky molecule, such as ethyl carbazate, would not cause sample precipitation. In fact, both spectra are similar except for the ethyl protons peak which is sharper and slightly shifted upfield in comparison to the tert-butyl peak (spectra not shown). The real oxidation degree was calculated by taking into account the ratio between the integral of the peak at $\delta 7.3 \mathrm{ppm}$ (arising from the carbazone group formed either with $\mathrm{tBC}$ or $\mathrm{EtC}$ ) and the integral of the anomeric proton at $\delta 4.9$ ppm. The oxidation degrees obtained with EtC were slightly higher (Table 1) than that obtained with $\mathrm{tBC}$ titration, which may suggest some difficulty of $\mathrm{tBC}$ to react further due to its bulkier moiety.

The dexOx number-average molecular weight $(\mathrm{Mn})$, estimated by a SEC analysis, showed a clear decrease with increasing oxidation degree, but also an increasing polidispersity index (PDI), reflecting the higher range of dexOx molecular weights (Table 1).

\subsection{DexOx solutions viscosity}

On the design of injectable hydrogel formulations, details, like viscosity characterization, become increasingly important, in order to choose the best syringe geometries and/or needle gauges. Generally, the hydrogel characteristics can be tailored by the feed concentration ${ }^{14}$, degree of polymer modification and in other cases, chemical initiators concentration ${ }^{24}$. This particular system involves the mixing of two equal-volume solutions, each of which, with its own reactive species, oxidized dextran residues and the dihydrazide. Thus, the formulations design has to take into account the feed concentration halving, of both solutions, after mixing. 
Every dexOx sample, at any of the concentrations tested, showed a linear shear stress increase regarding the different shear rates, hence, a Newtonian behavior. For every concentration tested, across the different OD's, the obtained viscosities for each dexOx series show a natural trend directly related with the concentration, as can be observed on Figure 2A for dextran and D40. The OD influence on viscosity seems to have a higher effect, when the polymer concentration is high, but as the polymer solution is less concentrated, the viscosity tends to decrease and eventually be lower than the reference solution (original dextran).

Within the same concentration range, only the D25 viscosity falls below the dextran viscosity, but on the other hand, D40 has an extremely high viscosity reflecting the influence of the high reactivity, due to the high OD (Figure 2B).

We suggest that this effect is due to the molecular weight decrease with oxidation, which, below a certain concentration, counter-balances the crosslinking via hemiacetals (Figure 1A), decreasing the solution viscosity (Figure 2A), which could possibly be further enhanced by a dexOx chain coil due to the intra hemiacetal formation (Figure 1B), demonstrating how both parameters (OD and Mw) can interfere on the viscosity of a given dexOx solution.

\subsection{Hydrogels characterization}

The hydrogels preparation took into consideration three factors: the dexOx concentration/oxidation degree and the AAD concentration. The first two are closely related due to the viscosity issues that were discussed above. Therefore, the concentration used was as high as the viscosity would allow it. Solutions of D5 and D10 with 30\% concentration allow a good homogenization, despite the high viscosity, but for D25, we had to use a $20 \%$ concentration. The D40 macromonomer was not studied in deep detail as the others, due to its high reactivity for the concentration range wanted. The D40 with a $20 \%$ concentration yields a very viscous solution that reacts promptly with AAD, impairing a good homogenization. Lower concentrations produce nicer hydrogels, however with less attractive and more unpredictable swelling profiles. Yet, the D40 gelation rate data is presented (15\% feed concentration), 
to strengthen the issue on the balance between $\mathrm{OD}$ and $\mathrm{AAD}$ feed concentration effect on the gelation rate, discussed below.

The balance between the available crosslinking points (oxidation degree) and the amount of crosslinker used (AAD feed concentration) clearly affects the gelation rate, as shown on Table 2, however, not always on the same direction. The gelation periods of D25 and D40 hydrogels decrease with the AAD increase, while for the D5 and D10 hydrogels, the gelation periods increase directly with the AAD concentration. When low OD's are present, the excess AAD increases the number of non-valid crosslinks, caused by the reaction of a single hydrazide, leaving a dangling end ${ }^{25}$, and retarding the hydrogel formation. The polymer feed concentration also affects the gelation periods, as can be observed with the D40 hydrogels, which should be faster than the D25, for the same AAD feed concentrations.

The dexOx/AAD hydrogels were prepared in Teflon molds and let to cure for at least two hours, except for the D5 hydrogels, which were left overnight, due to the slow curing rate. Equal volumes of dexOx and $\mathrm{AAD}$ solutions were added and mixed vigorously with the pipette tip, to achieve a good homogenization of both solutions.

The hydrazide groups in AAD react with the aldehyde groups in oxidized dextrans forming hydrazone bonds, which are hydrolysable, retrieving the same chemical groups (Figure 1C), therefore, making these hydrogels soluble in different time frames. The swelling index (Equation 1) of the different sets of hydrogels showed how the dissolution profiles could be controlled, not only by the $\mathrm{AAD}$ amount but as well by the dextran OD. By increasing the OD, we can significantly increase the dissolution time of the hydrogels. This oxidation increase, allows that more AAD can be used as a crosslinking agent, reflecting on more lasting hydrogels. By balancing these two variables it is possible to control the dissolution profile of the hydrogels.

As shown, low oxidation degrees do not allow long dissolution times. However, it is perfectly possible to control the dissolution profile with the AAD concentration, within a range of 5 days (Box in Figure 3). Rising up the oxidation, hydrogels more resistant to dissolution are obtained. The D25 
hydrogels have very interesting profiles (Figure 3). The dissolution time could be extended up to 70 days. Furthermore, one can observe an interesting plateau, with $20 \%$ AAD, resembling a saddle, approximately from the $2^{\text {nd }}$ until the $50^{\text {th }}$ day of immersion on phosphate buffer. Within this period the hydrogels maintained their size and shape without abrupt swelling variations. This profile seems to be interesting for the proposed application, as it will not swell above the injected volume and takes few weeks to dissolve. Just before the hydrogel collapses and dissolve, a maximum swelling peak is observed. We hypothesize this peak to be a fracture point, reached when the osmotic force equals the polymeric matrix force, which weakens through time, due to the AAD diffusion out of the hydrogel.

The water content of the vitreous humour is around $98 \%$, mainly composed of collagen, hyaluronic $\operatorname{acid}^{26}$ and hyalocytes of Balazs, which take care of removing cellular debris and reprocessing the hyaluronic $\operatorname{acid}^{27}$. Since the dexOx hydrogels are soluble in water and electrolyte solutions we think that the vitreous humour will naturally contribute to the dissolution of the hydrogel and help the hydrogel elimination. The by products of the designed hydrogel are its same initial components. Oxidized dextran and AAD. Both by-products should be excreted, to the aqueous humor, by the anterior route. This elimination is probably faster than through the blood-retina barrier to the systemic circulation $^{1}$, despite that dextrans, up to $150 \mathrm{kDa}$, are diffusible through the sclera ${ }^{28}$.

\subsection{Assessment of Cytotoxic Potential.}

The characteristics of these formulations render them suitable as a drug carrier to the posterior part of the eye, due to its injectable character, the controlled gelation rate or the dissolution profile shown. Formulations composed partially by dexOx have been tested for their biocompatibility. Recently, Bhatia and co-workers ${ }^{17}$, seeded $3 \mathrm{~T} 3$ fibroblast cells on top of oxidized dextran $(20 \%-50 \%$ OD) crosslinked with 8-arm PEG amine and showed that their formulation, was non-toxic and could lead to cell confluence after 24 hours of growth. This suggests, that the crosslinked-oxidized dextran can promote better cell adhesion than dextran itself ${ }^{29}$, despite showing some toxicity when present, alone, in cell culture with mesothelial cells ${ }^{30}$. 
In the present work, endothelial cells from rabbit cornea were chosen based on the need to assess the oxidized dextran AAD crosslinked hydrogels cytotoxicity for the proposed application.

To assess the cytotoxicity of these materials, several combinations of hydrogels involving the different dexOx's and AAD concentrations, were prepared. The 96-well cell culture plates were covered with $60 \mu \mathrm{L}$ of hydrogels or just controls and the cells were seeded on top, after an over-night curing period. The materials used were not only dissolved in PBS but also on the appropriate cell culture media. Occasionally, the initial dexOx feed concentration had to be slightly decreased, due to the high viscosity, probably due to medium proteins, that crosslink with the oxidized dextran through the existing amine groups.

After cell seeding on top of the hydrogels, each well was visualized in optical microscope, to observe whether there was any cell adhesion and/or proliferation. Cells grew in the presence of all hydrogels tested, although cell growth, in the presence of D25 hydrogels, did not achieve confluence during the period of study (Figure 4).

Dextran is neutrally charged and we expect dexOx to keep the neutrality, as the periodate oxidation reaction does not yield any charged chemical groups. However, the crosslinking of dexOx with AAD (pKa 2.5) yields hydrazone bonds which were reported to have a pKa in the order of 3 to $4^{31}$. Hence, due to the nature of the crosslinking bond, we suggest that the hydrogels zeta potential shifts negatively, allowing cell growth and proliferation.

It is well known that the surface chemistry of hydrogels can affect cell adhesion, proliferation and other phenomena. Chen et $\mathrm{al}^{32}$ work showed how the polymer nature and crosslinker concentration can dictate the surface charge density of the gels and strongly influence the cell behaviour. They have identified a threshold on the surface zeta potential $(-20 \mathrm{mV})$, below which cell adhere and proliferate.

Schneider and collaborators also reported that charge density of the hydrogel can regulate cell attachment either directly in the absence of extracellular matrix components, or indirectly through the association of extracellular matrix proteins found within the serum associated with charges of the hydrogel surface ${ }^{33}$. 
The MTS assay is a quick effective method for testing mitochondrial impairment and correlates quite well with cell proliferation. In recent years, it has been frequently used as a preliminary screen for the evaluation of in vitro cytotoxicity of polymeric components.

The MTS assays were performed at 1, 3 and 7 days after cells being seeded on top of the hydrogels. Cellular activity did not seem to be affected by the medium used to prepare the hydrogels (MEM or PBS). The results (Figure 5) emphasize that every formulation promoted dehydrogenase activity. In the first 24 hours, all hydrogels promoted higher enzyme activity than the negative control. By the $3^{\text {rd }}$ day, the activity doubled for most hydrogels, with a few exceptions, and by the $7^{\text {th }}$ day, the activity maintained the same levels. These results demonstrate the tested formulations as non-cytotoxic.

\section{Conclusions}

On the estimation of the dextran oxidation degree, we have identified ethyl carbazate as a better and more accurate titrant, than tert-butyl carbazate, especially for the higher oxidized samples. The bulkier tBC moiety causes sample precipitation and it also seems to impair good access to the oxidized residues lowering the measured oxidation degree.

The viscosity studies revealed how the dextran oxidation degree can limit the concentration used. However, for diluted dexOx solutions, the lower molecular weight, counter-balances the oxidation effect, decreasing the viscosity.

The characterized hydrogels have a dual crosslinking control method, which lies on the dextran OD and the amount of AAD used. The OD allows a given percentage of residues to serve as reactive points to molecules such as AAD. The amount of AAD used is crucial in defining the gelation period, mechanical properties and dissolution profile of the hydrogels. It should be within the same range of the amount of oxidized residues present in dextran, to avoid forming dangling ends, and not valid mechanical crosslinks.

On the cytotoxicity assay, the different dextran oxidation degrees tested were successful in promoting cell adhesion and growth. However, with the increasing oxidation, the cells took longer to 
proliferate in the hydrogels. Oddly, the metabolic activity, measured by the MTS assay, showed very high levels of activity for the higher oxidized hydrogels, not corresponding to the cellular proliferation observed.

The proposed and studied system aims at providing a sustainable drug delivery device to the posterior part of the eye, which requires a single injection, but does not require further surgery on the removal of the device. The designed formulation is expected to dissolve and be eliminated naturally by the organism.

\section{Acknowledgment}

The authors thank Graça Rasteiro for allowing the use of the viscometer and rheometer. This study was supported by Instituto de Investigação Interdisciplinar (financial support of JM - III/BIO/20/2005) and by the Portuguese Foundation for Science and Technology (in the form of fellowship to IJC SFRH/BPD/19776/2004).

\section{References}

[1] Del Amo EM, Urtti A. Current and future ophthalmic drug delivery systems. A shift to the posterior segment. Drug Discov Today 2008;13:135-143

[2] Schachar RA, Chen W, Woo BK, Pierscionek BK, Zhang X, Ma L. Diffusion of nanoparticles into the capsule and cortex of a crystalline lens. Nanotechnology 2008;19:1-4

[3] Urtti A. Challenges and obstacles of ocular pharmacokinetics and drug delivery. Adv Drug Deliv Rev 2006;58:1131-1135

[4] Le Bourlais C, Acar L, Zia H, Sado PA, Needham T, Leverge R. Ophthalmic drug delivery systems-recent advances. Progress in Retinal and Eye Research 1998;17:33-58 
[5] Hoffman AS. Hydrogels for biomedical applications. Adv Drug Deliv Rev 2002;54:3-12

[6] Van Tomme SR, Hennink WE, Biodegradable dextran hydrogels for protein delivery applications. Exp Rev Medical Dev 2007;4:147-164

[7] Ferreira LS, Gerecht S, Fuller J, Shieh HF, Vunjak-Novakovic G, Langer R, Bioactive hydrogel scaffolds for controllable vascular differentiation of human embryonic stem cells. Biomaterials $2007 ; 28: 2706-2717$

[8] Mehvar R, Dextrans for targeted and sustained delivery of therapeutic and imaging agents. J Control Rel 2000;69:1-25

[9] Jeanes A, Wilham CA, Periodate Oxidation of Dextran. J. Am. Chem. Soc. 1950;72:2655-2657

[10] Suvorova OB, Iozep AA, Passet BV, Reactivity of Polysaccharide Aldehydes toward NNucleophiles. Russian J App Chem 2001;74:1016-1020

[11] Domb AJ, Linden G, Polacheck I, Benita S, Nystatin-dextran conjugates: Synthesis and characterization. J Polym Sci Pol Chem 1996;34:1229-1236

[12] Penzol G, Armisen P, Fernandez-Lafuente R, Rodes L, Guisan JM, Use of dextrans as long and hydrophilic spacer arms to improve the performance of immobilized proteins acting on macromolecules. Biotechnol Bioeng 1998;60:518-523

[13] Draye JP, Delaey B, Van de Voorde A, Van Den Bulcke A, Bogdanov B, Schacht E, In vitro release characteristics of bioactive molecules from dextran dialdehyde cross-linked gelatin hydrogel films. Biomaterials 1998;19:99-107

[14] Weng L, Chen X, Chen WC, Rheological characterization of in situ crosslinkable hydrogels formulated from oxidized dextran and N-carboxyethyl chitosan. Biomacromolecules 2007;8:11091115 
[15] Weng L, Romanov A, Rooney J, Chen WC, Non-cytotoxic, in situ gelable hydrogels composed of N-carboxyethyl chitosan and oxidized dextran. Biomaterials 2008;29:3905-3913

[16] Schacht E, Bogdanov B, Bulcke AVD, De Rooze N, Hydrogels prepared by crosslinking of gelatin with dextran dialdehyde. React Funct Polymers 1997;33:109-116

[17] Bhatia SK, Arthur SD, Chenault HK, Kodokian GK, Interactions of polysaccharide-based tissue adhesives with clinically relevant fibroblast and macrophage cell lines. Biotechnol Lett 2007;29:16451649

[18] Heindel ND, Zhao H, Leiby J, VanDongen JM, Lacey CJ, Lima DA, Shabsoug B, Buzby JH, Hydrazide Pharmaceuticals as Conjugates to Polyaldehyde Dextran: Syntheses, Characterization, and Stability. Bioconjug Chem 1990;1:77-82

[19] Maia J, Ferreira L, Carvalho R, Ramos MA, Gil MH, Synthesis and characterization of new injectable and degradable dextran-based hydrogels. Polymer 2005;46:9604-9614

[20] Bouhadir KH, Hausman DS, Mooney DJ, Synthesis of cross-linked poly(aldehyde guluronate) hydrogels. Polymer 1999;40:3575-3584

[21] Jia XQ, Burdick JA, Kobler J, Clifton RJ, Rosowski JJ, Zeitels SM, Langer R, Synthesis and characterization of in situ cross-linkable hyaluronic acid-based hydrogels with potential application for vocal fold regeneration. Macromolecules 2004;37:3239-3248

[22] Natu MV, Sardinha JP, Correia IJ, Gil MHG, Controlled release gelatin hydrogels and lyophilisates with potential application as ocular inserts. Biomed Mater 2007;2:241-249

[23] MacCallum DK, Lillie JH, Scaletta LJ, Occhino JC, Frederick WG, Ledbetter SR, Bovine corneal endothelium in vitro. Elaboration and organization and of a basement membrane. Exp Cell Res 1982;139:1-13 
[24] Vervoort L, Vinckier I, Moldenaers P, Van den Mooter G, Augustijns P, Kinget R. Inulin hydrogels as carriers for colonic drug targeting. Rheological characterization of the hydrogel formation and the hydrogel network. J Pharm Sci 1999;88:209-214

[25] Lee KY, Bouhadir KHB, Mooney DJM, Degradation Behavior of Covalently Cross-Linked Poly(aldehyde guluronate) Hydrogels. Macromolecules;2000 33:97-101

[26] Chirile TV, Hong Y. The Vitreous Humor. In Black J, Hastings GW, editors. Handbook of Biomaterials Properties. London: Chapman \& Hall; 1998. p 125-134

[27] Grabner G, Boltz G, Förster O. Macrophage-like properties of human hyalocytes. Invest Ophth Vis Sci 1980;19:333-340

[28] Ambati J et al. Diffusion of high molecular weight compounds through sclera. Invest Ophth Vis Sci 2000;41:1181-1185

[29] Massia SP, Stark J, Letbetter DS, Surface-immobilized dextran limits cell adhesion and spreading. Biomaterials 2000;21:2253-2261

[30] Ito T, Yeo Y, Highley CB, Bellas E, Kohane DS, Dextran-based in situ cross-linked injectable hydrogels to prevent peritoneal adhesions. Biomaterials 2007;28:3418-3426

[31] Rando RR, Orr GA, Bangerter FW, Throshold effects on the concanavalin-A-mediated agglutination of modified erythrocytes. J Biol Chem 1979;254:8318-8323

[32] Chen YM, Shiraishi N, Satokawa H, Kakugo A, Narita T, Gong JP, Osada Y, Yamamoto K, Ando J, Cultivation of endothelial cells on adhesive protein-free synthetic polymer gels. Biomaterials $2005 ; 26: 4588-4596$

[33] Schneider GB, English A, Abraham M, Zaharias R, Stanford C, Keller J, The effect of hydrogel charge density on cell attachment. Biomaterials 2004;25:3023-3028 


\section{Figure Captions}

Figure 1 - The reactive aldehyde groups formed upon periodate oxidation are prone to establish inter (A) or intra (B) hemiacetals, when reacting with hydroxyl groups from nearby residues. The addition of AAD (C) promotes the reversible crosslinking with the formation of hydrazones.

Figure 2 - Shear stress versus Shear rate. (A) - Dextran with concentrations (w/w) of: $\square-30 \%, \bigcirc$ $20 \%, \triangle-15 \%$ and $\diamond-10 \%$; and D40 with concentrations (w/w) of: $-20 \%, \boldsymbol{\Delta}-15 \%$ and 10\%. (B) All solutions at 20\% (w/w) $\bigcirc$ - dextran, $\square$ - D5, $\diamond-\mathrm{D} 10, \triangle-\mathrm{D} 25$ and $\nabla-\mathrm{D} 40$

Figure 3 - Swelling index profile of dexOx + AAD hydrogels. D25 with feed concentration of 20\% and D10 and D5 with feed concentration of 30\%. $\diamond-$ D25A5; $\diamond-$ D25A10; $\nabla-$ D25A20; Sub-view: D10A5; O -D10A10; $\triangle$ - D10A20; $\mathbf{- D 5 A 5 ;} \bullet$ - D5A10.

Figure 4 - Endothelial cells isolated from rabbit cornea seeded in dexOx+AAD hydrogels, dissolved in MEM or PBS, after 72h. A - D5A5, PBS; B - D5A5, MEM; C - D10A10, PBS; D - D10A10, MEM; E - D25A10, PBS; F - D25A10, MEM; G - Negative control; H - Positive control. Original Magnification X100.

Figure 5 - Cellular activities measured by the MTS assay after 1 day (open bars), 3 days (grey bars) and 7 days (black bars). Endothelial cells from rabbit cornea, seeded onto dexOx+AAD hydrogels. The materials were either dissolved in PBS or MEM. K+: Positive control; K-: Negative control. All error bars represent one standard deviation from six experiments. 


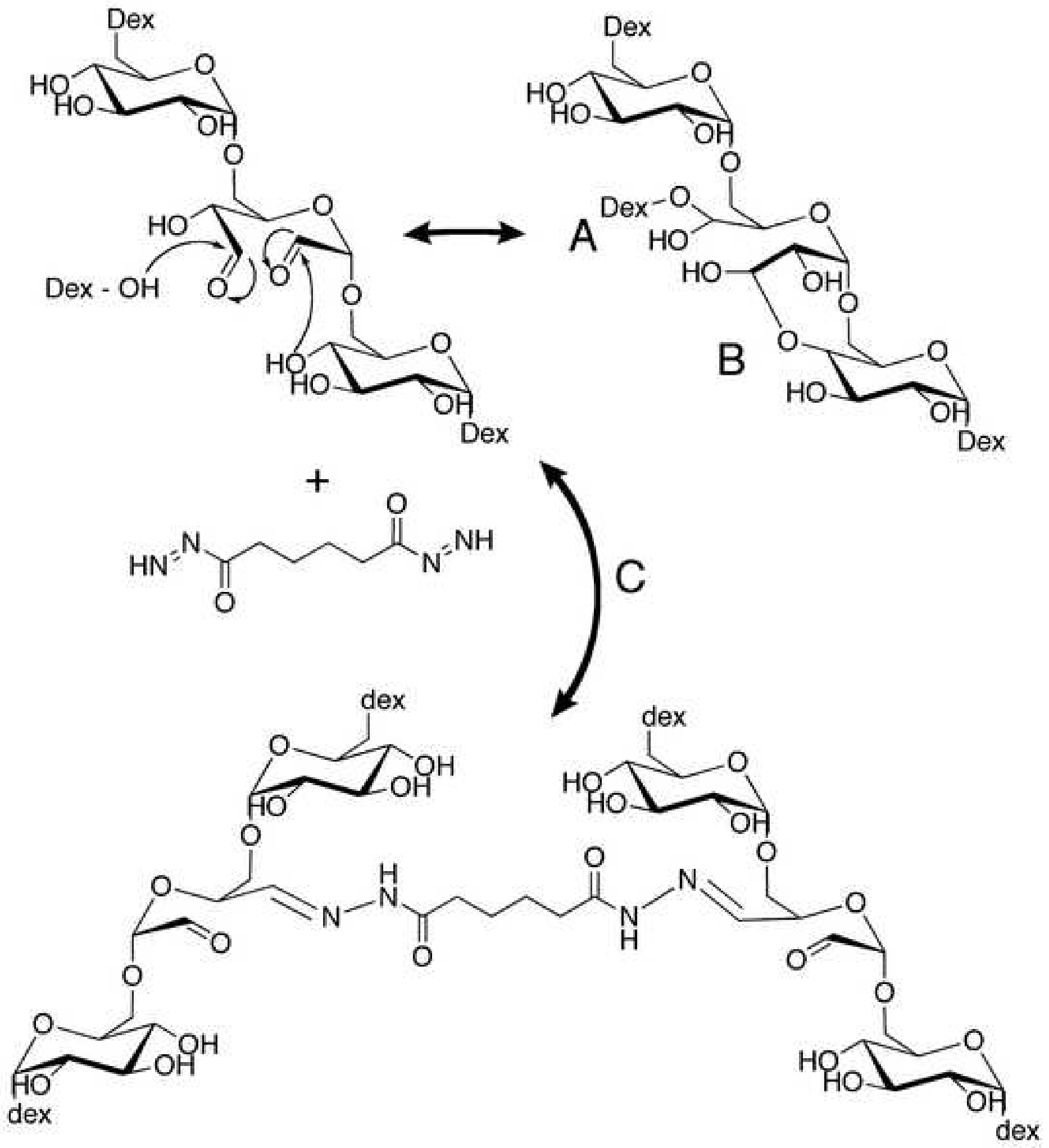




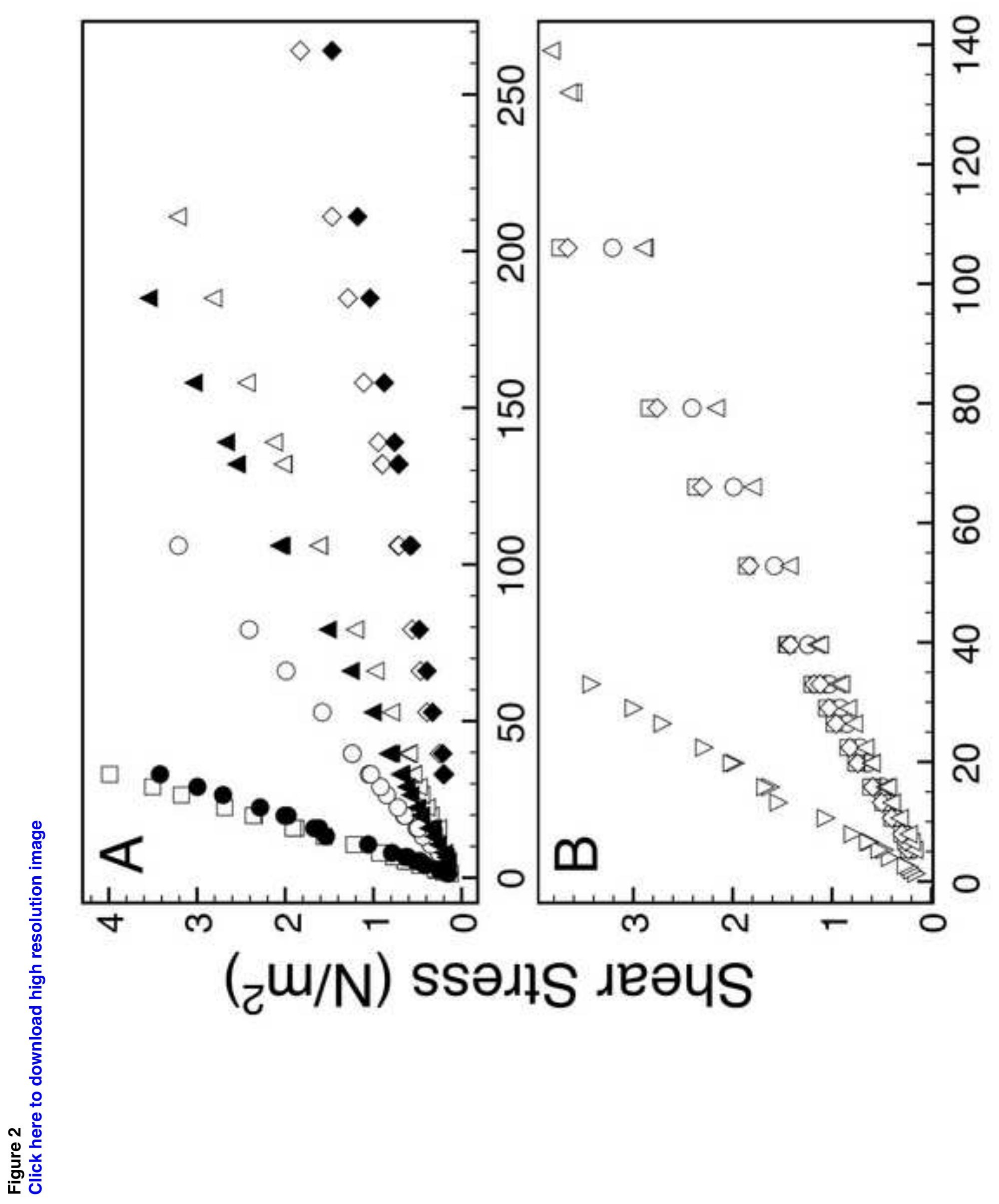




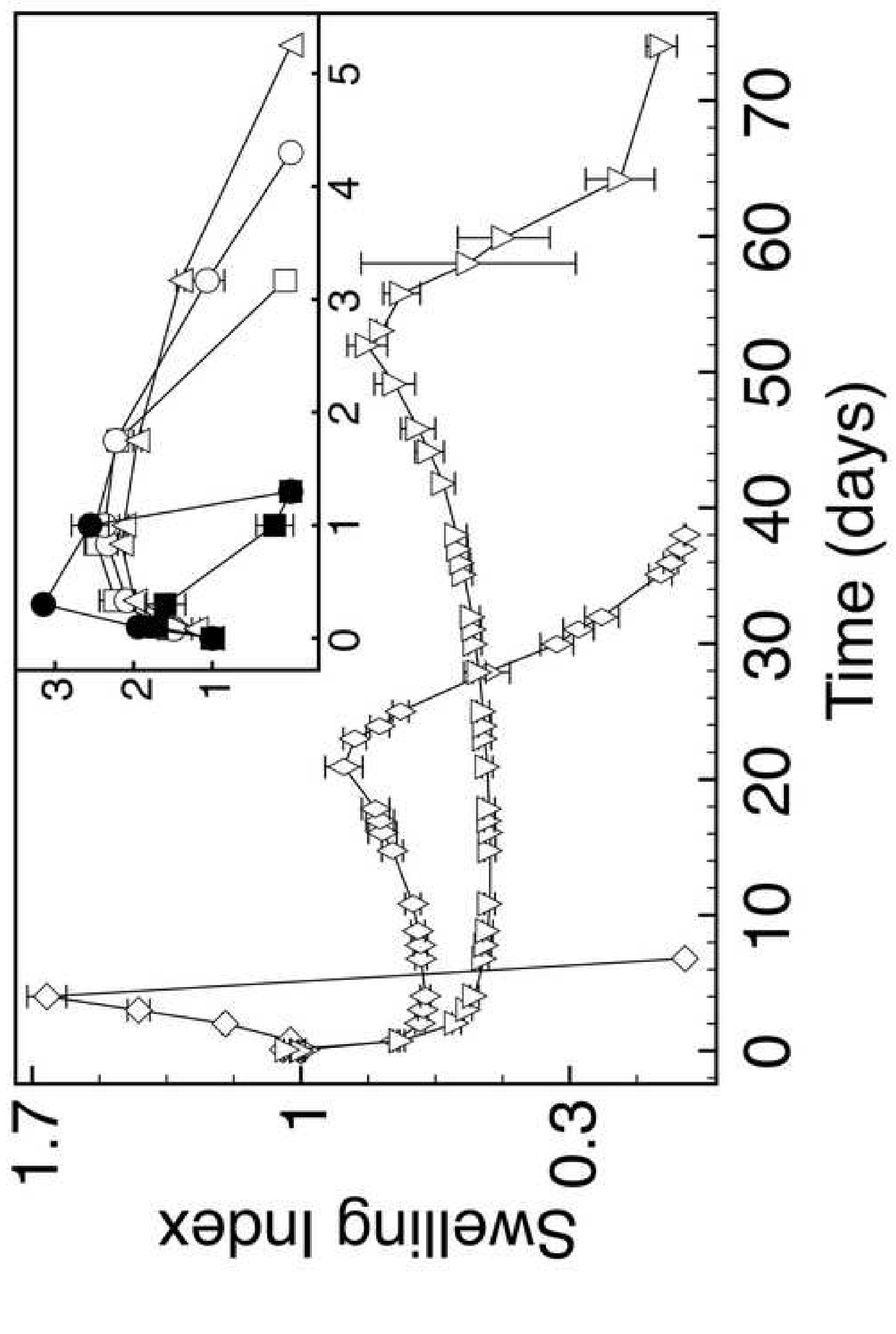




\section{Figure 4}

Click here to download high resolution image
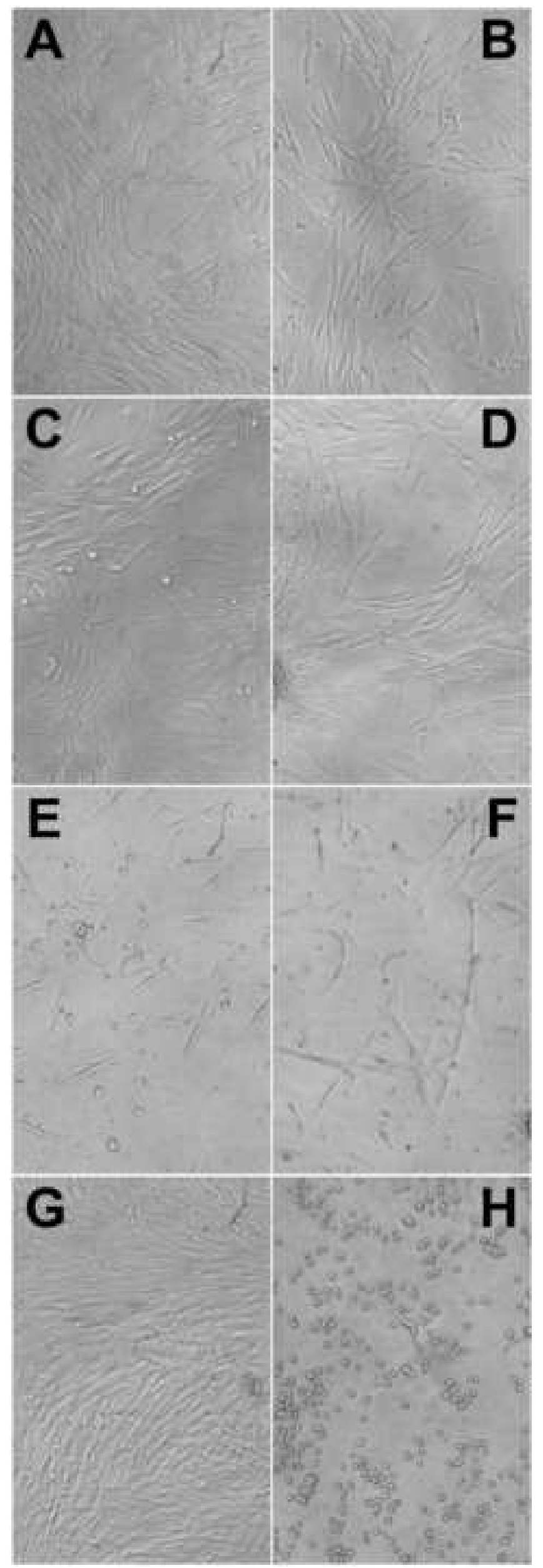
ก

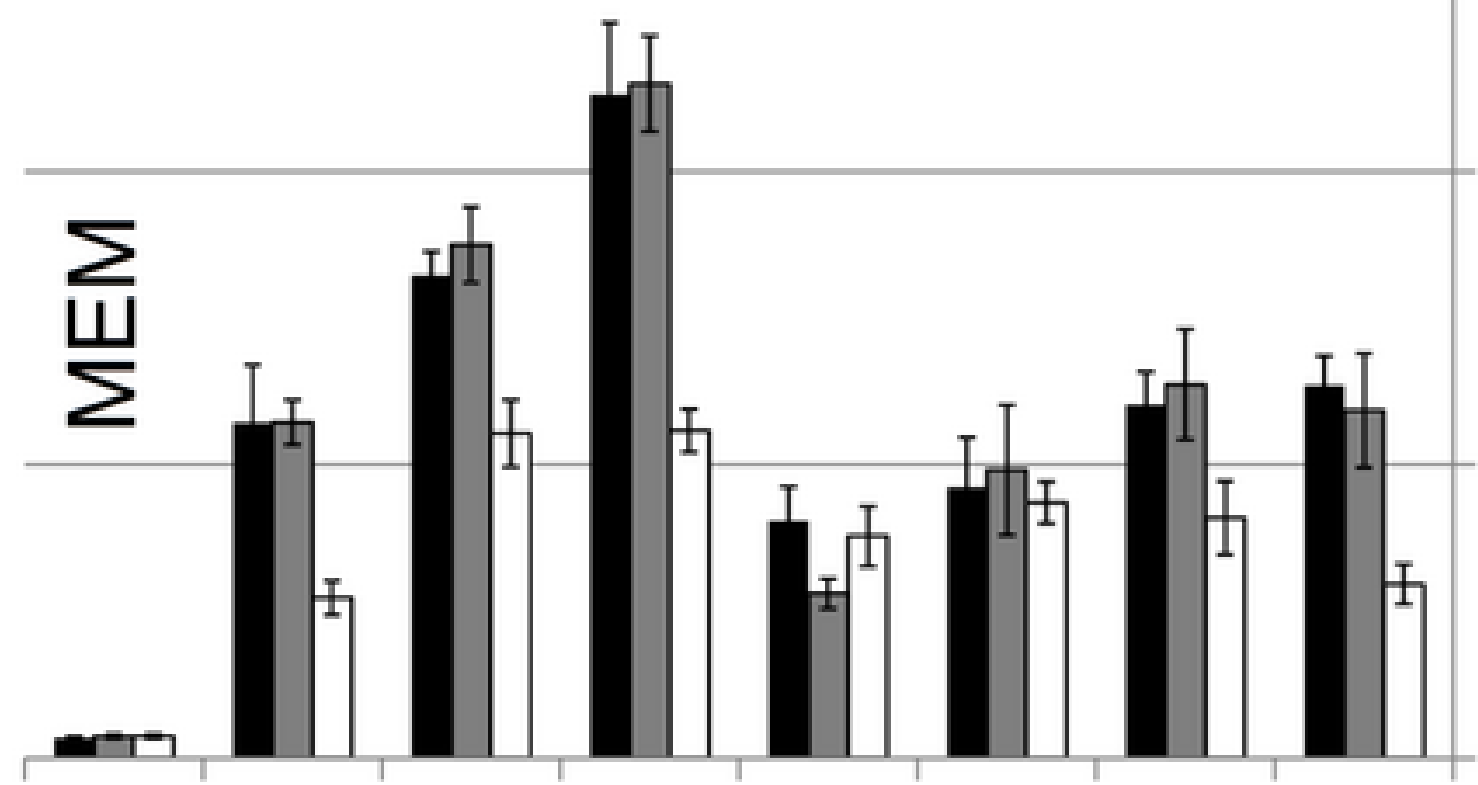

๑

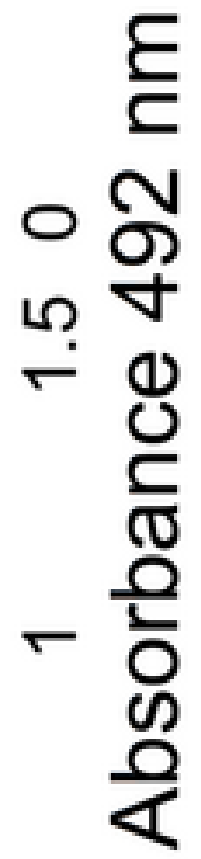

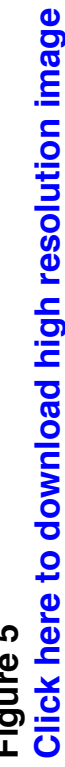

I

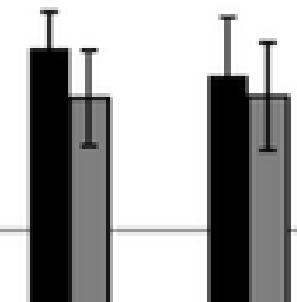

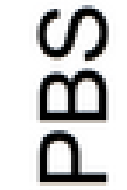
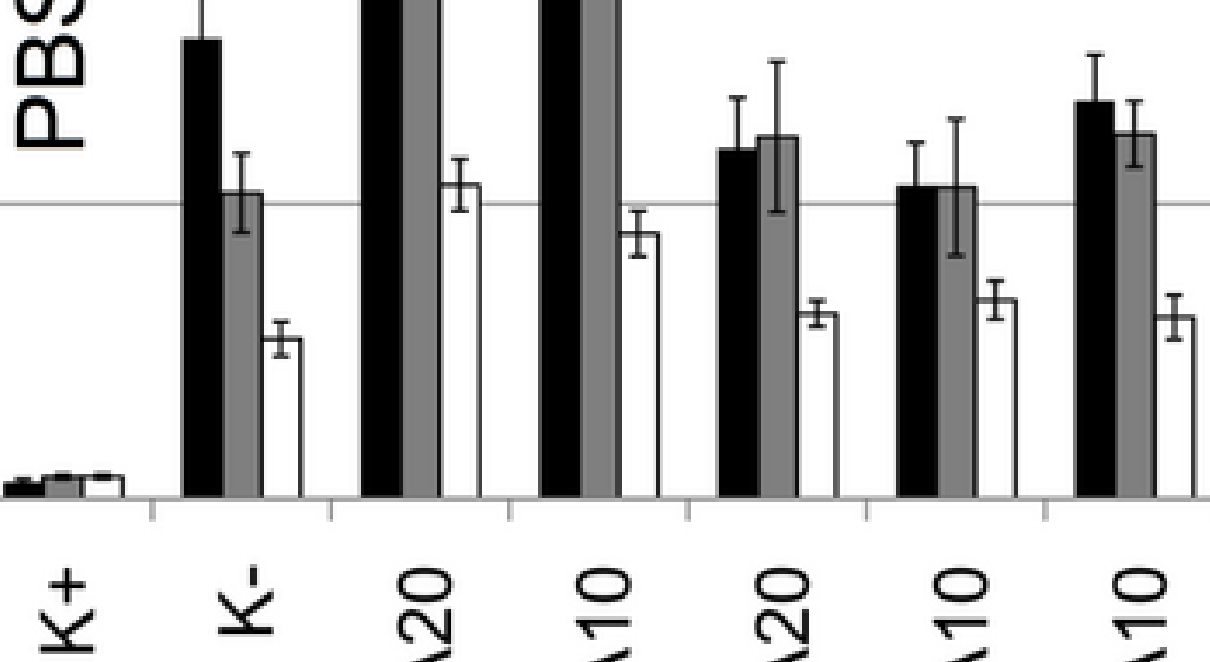

甶

?

I

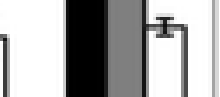

$\Gamma$

0 On

$\Omega \vee$

(1)

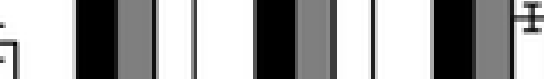


Tables: Maia et al

Table 1 - Oxidation Degree of several oxidized dextrans calculated by ${ }^{1} \mathrm{H}-\mathrm{NMR}$ analysis after titration with different carbazates and molecular weight evolution.

\begin{tabular}{cccccc}
\hline \multirow{2}{*}{ Sample } & \multicolumn{3}{c}{ Oxidation degree } & \multirow{2}{*}{$\mathrm{Mn}^{\mathrm{c}}$} & PDI $^{\mathrm{d}}$ \\
\cline { 2 - 4 } & $\mathrm{NaIO}_{4}{ }^{\mathrm{a}}$ & $\mathrm{tBC}^{\mathrm{b}}$ & $\mathrm{EtC}^{\mathrm{b}}$ & & \\
\hline Dextran & - & - & - & 40.9 & 1.47 \\
\hline $\mathrm{D} 5$ & 5 & $2.5 \pm 0.3$ & $3.6 \pm 0.1$ & 40.8 & 1.59 \\
\hline $\mathrm{D} 10$ & 10 & $7.4 \pm 0.9$ & $8.6 \pm 0.2$ & 37.9 & 1.61 \\
\hline $\mathrm{D} 25$ & 25 & $18.9 \pm 0.4$ & $22.2 \pm 0.7$ & 29.7 & 2.03 \\
\hline $\mathrm{D} 40$ & 40 & $-{ }^{\mathrm{e}}$ & $33.0 \pm 0.8$ & 8.3 & 3.05
\end{tabular}

${ }^{\text {a }}$ Theoretical OD, calculated as the molar ratio of sodium periodate per initial glucose unit in dextran.

${ }^{\mathrm{b}}$ Calculated by ${ }^{1} \mathrm{H}-\mathrm{NMR}$ after titration with tert-butyl carbazate/ethyl carbazate, taking into account the ratio between the integral of the peak at $\delta 7.3 \mathrm{ppm}$ and the integral of the anomeric proton at $\delta 4.9$ ppm. Average and standard deviation of 5 independent integrations.

${ }^{\mathrm{c}}$ Number-average molecular weight estimated by SEC

${ }^{\mathrm{d}}$ Polydispersity index corresponding to $\mathrm{Mw} / \mathrm{Mn}$

${ }^{\mathrm{e}}$ Precipitation occurred. 
Table 2 - Gelation periods estimated for each dexOx with different AAD concentrations.

\begin{tabular}{ccccc}
\hline \multirow{2}{*}{$\operatorname{dexOx}$} & $\begin{array}{c}\text { Feed conc. } \\
\%(\mathrm{w} / \mathrm{w})\end{array}$ & $5 \%$ AAD & $10 \%$ AAD & $20 \%$ AAD \\
\cline { 3 - 5 } & 30 & $66.7 \pm 1.4$ & $77.8 \pm 5.2$ & ${ }^{\mathrm{b}}$ \\
\hline $\mathrm{D} 5$ & 30 & $14.9 \pm 1.7$ & $16.8 \pm 0.5$ & $18.8 \pm 1.3$ \\
\hline $\mathrm{D} 10$ & 20 & $2.8 \pm 0.3$ & $1.6 \pm 0.9$ & $1.3 \pm 0.2$ \\
\hline $\mathrm{D} 25$ & 15 & $3.7 \pm 0.4$ & $2.6 \pm 0.1$ & $2.3 \pm 0.1$ \\
\hline D40 & & & & \\
\hline
\end{tabular}

a The gelation period was considered when $G^{\prime}=G^{\prime}$; values are mean $\pm \operatorname{SD}(\mathrm{n}=4)$.

${ }^{\mathrm{b}}$ Hydrogel not formed. 